Ann. Biol. anim. Bioch. Biophys., I972, 12 (4), 66I-665.

NOTE

\title{
UTILISATION D'UN DIAGNOSTIC PRÉCOCE DE GESTATION PAR ESTIMATION DU TAUX DE PROGESTÉRONE DANS LE SANG PÉRIPHÉRIQUE DE BREBIS
}

\author{
J. SAUMANDE et J. THIMONIER \\ avec la collaboration technique de Mile Nadine Gonzalìs D. ANDRÉ et C. Cornu \\ Station de Recherches sur la Physiologie de la Reproduction, I. N. R. A., \\ Centre de Recherches de Tours, I. N. R. A., \\ 37380 Nouzilly
}

\section{INTRODUCTION}

Chez les Ovins, 1'établissement de la gestation est possible, quel que soit le moment de 1'année (THIMONIER et CoGNIE, I97I), mais une discrimination précoce entre les femelles gestantes et celles qui ne le sont pas n'est pas toujours réalisable. En effet, pendant 1'anœstrus post-partum ou l'anœestrus saisonnier chez les brebis et en toutes saisons chez les agnelles, l'absence de retour en cestrus n'est pas obligatoirement liée à un état de gestation.

BASSETT et al. (I969) ont proposé d'utiliser le dosage de la progestérone comme test de la gestation chez la brebis. En I97I, ROBERTSON et SARDA ont montré le bien-fondé de cette idée. Nous avons cherché à appliquer ce test comme complément aux techniques modernes de reproduction, notamment après synchronisation des cycles sexuels. Nous l'avons comparé à deux autres méthodes de diagnostic de gestation.

\section{MATÉRIEL ET MÉTHODES}

\section{A. - Animaux}

L'expérience a été réalisée d'une part, au début de l'anœestrus saisonnier sur 30 femelles Préalpes adultes à la fin de la période d'allaitement et, d'autre part, pendant la saison sexuelle sur 49 agnelles Ile-de-France âgées de 7 à 8 mois. Dans les deux cas, les animaux ont été inséminés systématiquement, indépendamment de la venue en œstrus, 48 et $60 \mathrm{~h}$ après l'arrêt d'un traitement progestatif associé à PMSG (CoLAS et al., I97I). 


\section{B. - Techniques de diagnostic de gestation}

Plusieurs méthodes ont été employées :

- Détection de l'œestrus une fois par jour du $16^{e}$ au $19^{e}$ jour ou du $\mathrm{r}^{\mathrm{e}}$ au $20^{\mathrm{e}}$ jour après insémination artificielle. L'absence de retour en ostrus a été considérée comme caractéristique d'un état de gestation.

- Examen celioscopique du tractus génital (Thimonier et MaUléon, 1969) : le maintien des corps jaunes le $\mathrm{I} 8^{\mathrm{e}}$ jour après insémination artificielle chez les agnelles Ile-de-France ou la présence d'un utérus distendu et irrigué 40 jours après insémination artificielle chez les brebis Préalpes ont été tenus pour caractéristiques du début de la gestation.

- Estimation du taux de progestérone dans le plasma ou le sérum du sang périphérique I6 ou I7 jours après insémination artificielle. Le taux de progestérone a été apprécié selon une méthode de compétition de liaison à une protéine. Pour chaque animal, un seul échantillon a été traité. Un millilitre de plasma a été extrait une fois par $5 \mathrm{ml}$ d'hexane Prolabo RP (récupération : 83,4 p. I00 $\pm 0,8 ; n=\mathrm{I} 2$ ). Après évaporation, $0,5 \mathrm{ml}$ d'une solution contenant du plasma de chien et de la corticostérone tritiée a été ajouté à chaque échantillon. Ensuite, la méthode est identique à celle décrite par BASSETT et al., r 969. Cependant, contrairement aux auteurs précédemment cités, nous n'avons pas calculé le taux de progestérone : il est alors suffisant de compter la radioactivité liée pendant un temps très court soit $20 /$ Ioo de minute dans un compteur à scintillation liquide Packard Tricarb 3375. Lorsque le nombre de cpm liés est inférieur à celui du standard de I ng, l'animal, dont provient l'échantillon, est considéré comme gestant.

\section{RÉSULTATS ETT DISCUSSION}

Les diagnostics effectués ont été comparés aux mises bas. Les résultats sont présentés dans les tableaux I et 2 .

TABIEAU I

Comparaison de l'exactitude de différentes techniques de diagnostic de gestation chez les brebis de race Préalpes

\begin{tabular}{|c|c|c|c|c|}
\hline \multicolumn{2}{|c|}{ Technique de diagnostic } & $\begin{array}{l}\text { Détection de } \\
\text { l'œstrus }\end{array}$ & $\begin{array}{c}\text { Estimation } \\
\text { Progestérone } \\
\text { (Plasma) }\end{array}$ & Endoscopie \\
\hline \multicolumn{2}{|c|}{ Moment d'application } & $\mathrm{J}_{16}$ à $\mathrm{J}_{19}\left(^{2}\right)$ & $\mathrm{J}_{16}$ & $\mathrm{~J}_{40}$ \\
\hline \multirow{2}{*}{$\begin{array}{c}\text { Femelles } \\
\text { diagnostiquées } \\
\text { gestantes }\end{array}$} & Nombre & 22 & 16 & 14 \\
\hline & $\begin{array}{c}\text { P. } 100 \\
\text { diagnostics } \\
\text { exacts }\end{array}$ & 50 & 68,8 & 78,6 \\
\hline \multirow{2}{*}{$\begin{array}{c}\text { Femelles } \\
\text { diagnostiquées } \\
\text { non gestantes }\end{array}$} & Nombre & 8 & 14 & 16 \\
\hline & $\begin{array}{c}\text { P. } 100 \\
\text { diagnostics } \\
\text { exacts }\end{array}$ & 100 & 100 & 100 \\
\hline \multicolumn{2}{|c|}{ Exactitude p. $100(1)$} & 63,3 & 83,3 & 90,0 \\
\hline
\end{tabular}

(1) Exactitude $=\frac{\text { Nombre total de diagnostics exacts }}{\text { Nombre total de diagnostics }} \times 100$.

(2) $\mathrm{J}_{0}=$ Jour de l'insémination artificielle. 
Dans les conditions de 1'expérience, il apparaît que, à l'exception de la détection de l'œstrus, les techniques étudiées permettent un diagnostic très précoce avec une grande exactitude. Quelles que soient les méthodes, les animaux diagnostiqués non gestants n'ont jamais mis bas. La différence entre les mises bas et les diagnostics positifs effectués par appréciation du taux de progestérone ou par endoscopie, peut être due, soit à des erreurs de diagnostic, soit à l'existence d'une mortalité embryonnaire (VANDEPLASSCHE, I968). Le fait que, chez les brebis Préalpes, l'exactitude du diagnostic de gestation par endoscopie à $\mathrm{J}_{40}$ soit supérieure à celui porté à $\mathrm{J}_{16}$ par appréciation du taux de progestérone, alors que chez les agnelles Ile-de-France, les deux techniques donnent les résultats identiques, est en faveur de la deuxième hypothèse.

\section{TABLEAU 2}

Comparaison de l'exactitude de différentes techniques de diagnostic de gestation chez les agnelles de race Ile-de-France

\begin{tabular}{|c|c|c|c|c|c|}
\hline \multirow{2}{*}{\multicolumn{2}{|c|}{ Technique de diagnostic }} & \multirow{2}{*}{$\begin{array}{l}\text { Détection de } \\
\text { l'œstrus }\end{array}$} & \multicolumn{2}{|c|}{ Estimation Progestérone } & \multirow{2}{*}{ Endoscopie } \\
\hline & & & Plasma & Sérum & \\
\hline \multicolumn{2}{|c|}{ Moment d'application } & $\mathrm{J}_{13}$ à $\mathrm{J}_{20}\left(^{(2)}\right.$ & $\mathrm{J}_{17}$ & $\mathrm{~J}_{17}$ & $\mathrm{~J}_{18}$ \\
\hline \multirow{2}{*}{$\begin{array}{c}\text { Femelles } \\
\text { diagnostiquées } \\
\text { gestantes }\end{array}$} & Nombre & 34 & 26 & 25 & 25 \\
\hline & $\begin{array}{c}\text { P. } 100 \\
\text { diagnostics } \\
\text { exacts }\end{array}$ & 70,6 & 92,3 & 92,0 & 92,0 \\
\hline \multirow{2}{*}{$\begin{array}{c}\text { Femelles } \\
\text { diagnostiquees } \\
\text { non gestantes }\end{array}$} & Nombre & 15 & 23 & 24 & 22 \\
\hline & $\begin{array}{l}\text { P. } 100 \\
\text { diagnostics } \\
\text { exacts }\end{array}$ & 100 & 100 & 95,8 & 100 \\
\hline \multicolumn{2}{|c|}{ Exactitude P. $100\left({ }^{1}\right)$} & 79,6 & 95,9 & 93,9 & 95,7 \\
\hline
\end{tabular}

(1) Exactitude $=\frac{\text { Nombre total de diagnostics exacts }}{\text { Nombre total de diagnostics }} \times 100$.

$\left({ }^{2}\right) \mathrm{J}_{\mathbf{0}}=$ Jour de l'insémination artificielle.

L'absence de retour en cestrus chez certains animaux non gestants, que ce soit chez les agnelles Ile-de-France pendant la période normale de reproduction ou chez les brebis adultes au début de l'anœstrus saisonnier, est un phénomène déjà décrit (THIMONIER et al., I968) et rend compte de la différence d'exactitude entre l'étude du retour en œstrus et les deux autres techniques étudiées.

La technique d'endoscopie utilisée, plus rapide ( 2 minutes d'observation par animal en moyenne), réalisée plus tôt, aussi exacte que celle décrite par PHILIPPo et al. (I97I) reste malgré tout une intervention chirurgicale ; elle est pour cela à déconseiller comme technique de routine sauf si l'on veut connaître avec précision le nombre d'ovulations. 
La méthode d'appréciation du taux de progestérone périphérique utilisée dans cette étude permet d'obtenir une exactitude identique à celle publiée par ROBERTSON et SARDA (I97I). Deux personnes peuvent réaliser 700 à 800 tests par semaine, soit 4 fois plus que les auteurs précédemment cités.

Cette technique est le complément indispensable à toutes celles qui ont été déjà proposées (synchronisation de l'œestrus et insémination artificielle) pour l'intensification de la production de l'élevage ovin. La possibilité de réaliser le diagnostic de gestation sur du sérum, celle de prélever les échantillons indifféremment le $16^{\mathrm{e}}, \mathrm{I} 7^{\mathrm{e}}$, I $8^{\mathrm{e}}$ ou I9 $\mathrm{I}^{\mathrm{e}}$ jour après insémination artificielle (travail en cours) lui donne une grande souplesse d'utilisation et permet d'espérer son développement.

Ręu pour publication en juin 1972.

\section{REMERCIEMENTS}

Les auteurs remercient monsieur COGNIE qui a effectué les prélèvements de sang et réalisé l'examen cœlioscopique quarante jours après l'insémination artificielle des brebis.

\section{SUMMARY}

UTILIZATION OF AN EARLY PREGNANCY TEST BY PROGESTERONE

ASSAY IN THE PERIPHERAL BLOOD IN THE EWE

An early pregnancy test ( 16 th and 17 th days after artificial insemination) by progesterone assay in the peripheral blood plasma or serum has been tested in adult ewes at the beginning of the seasonal anestrus period and in maiden ewes during the breeding season. A simplified competitive protein binding method was used : 700 to 800 pregnancy diagnosis can be performed weekly by two technicians with an accuracy of $83,3 \mathrm{p}$. roo and $92,5 \mathrm{p}$. I oo respectively in the anestrus period and during the breeding season. The results obtained with this method are compared with those tabulated from non-returns and endoscopic ovarian examinations.

\section{RÉFÉRENCES BIBLIOGRAPHIQUES}

Bassett J. M., Oxborrow T. J., Smith I. D., Thorburn G. D., Ig69. The concentration of progesterone in the peripheral plasma of the pregnant ewe. J. Endocr., 45, 449-457.

Colas G., Brice G., Courot M., Cotrier M., rg7i. L'insémination artificielle dans les plans d'intensification de la production ovine : état actuel et perspectives. Bull. Tech. Inform. Minist. Agric., 257, I47-I52.

Phillippo M., Swapp G. H., Robinson J. J., Gill J. C., I97I. The diagnosis of pregnancy and estimation of fotal numbers in sheep by laparoscopy. J. Reprod. Fert., 27, 129-132.

Robertson H. A., SARda I. R., i97I. A very early pregnancy test for mammals : its application to the cow, ewe and sow. J. Endocr., 49, 407-419.

Thimonier J., Cognie Y., r97r. Accélération des mises bas et conduite d'élevage chez les bovins. Bull. Tech. Inform. Minist. A gric., 257, 187-196. 
Thimonier J., Mautéon P., r969. Variations saisonnières du comportement d'œstrus et des activités ovarienne et hypophysaire chez les ovins. Ann. Biol. anim. Bioch. Biophys., 9, 233-250.

Thimonier J., Mauléon P., Cognie Y., Ortavant R., I968. Déclenchement de l'œestrus et obtention précoce de gestations chez des agnelles à l'aide d'éponges vaginales imprégnées d'acétate de fluorogestone. Ann. Zootech., 17, 275-288.

VANDEPLASSChe M., Ig68. La mortalité embryonnaire et son diagnostic VIe Cong. Intern. Reprod. Anim. Insem. Artif., Paris, vol. 1, 347-39r. 